\title{
Estudio material por FTIR-ATR y examen morfológico para el uso de tubos de encofrado como soporte de almacenaje de tapices
}

\author{
María López Rey, Ruth Chércoles Asensio
}

\begin{abstract}
Resumen: Actualmente, se recomienda el almacenamiento de los tapices enrollados sobre un soporte cilíndrico. Los cilindros ideales están fabricados con cartón libre de ácido o polietileno de alta densidad, sin embargo el alto precio y la escasa comercialización de los mismos hace que sea frecuente recurrir a materiales de construcción, aunque es necesario comprobar su compatibilidad con los tapices, y para ello se debe conocer su composición química y sus propiedades físicas.

En este trabajo se estudian las propiedades químicas de componentes mayoritarios por espectroscopia de infrarrojos por transformada de Fourier con dispositivo ATR acoplado (FTIR-ATR), análisis elemental con microscopía electrónica de barrido con energías dispersivas de rayos X (MEB-EDX) y examen morfológico por técnicas microscópicas de tubos de encofrado como solución ofrecida por la industria de la construcción para su utilización como soporte de almacenaje para tapices. Además, se muestra un ejemplo práctico de uso, empleándolos para el almacenaje de la colección de Tapices de Carmen y Justo Fernández, conservada en la Biblioteca Histórica Marqués de Valdecilla de la Universidad Complutense.
\end{abstract}

Palabras clave: Tapices, almacenaje, conservación preventiva, FTIR-ATR, análisis físico-químico

\section{Material study by FTIR-ATR and morphological examination for the use of formwork pipes as storage support for tapestries}

Abstract: Nowadays, it is recommended to store tapestries rolled up on a cylindrical support. The ideal cylinders are made of acid-free cardboard or high-density polyethylene. However, the high price and its poor marketing, makes it common to resort to construction materials. To verify their compatibility with tapestries, it is necessary to know their chemical composition and physical properties.

In this work, the chemical properties of major components are studied by Fourier transform infrared spectroscopy coupled with ATR device (FTIR-ATR), elemental analysis with scanning electron microscopy (SEM-EDS) and morphological examination by microscopic techniques of formwork tubes as a solution offered by the construction industry for it use as a storage support for tapestries. In addition, an example using the tubes for the storage of the tapestry collection, Carmen y Justo Fernández, preserved in the Biblioteca Histórica Marqués de Valdecilla de la Universidad Complutense.

Keywords: Tapestry, storage, preventive conservation, ATR-FTIR, physical-chemical analysis

\section{Estudo material por FTIR-ATR e exame morfológico para o uso de tubos de cofragem como suporte de armazenamento de tapeçarias}

Resumo: Atualmente, recomenda-se o armazenamento das tapeçarias enroladas sobre um suporte cilíndrico. Os cilindros ideais são fabricados com cartão livre de ácido ou polietileno de alta densidade, porém o alto preço e a escassa comercialização dos mesmos faz com que seja frequente recorrer a materiais de construção, embora seja necessário verificar a sua compatibilidade com as tapeçarias, e para isso deve-se conhecer a sua composição química e as suas propriedades físicas.

Neste trabalho estudam-se as propriedades químicas de componentes maioritários por espectroscopia de infravermelhos por transformada de Fourier com dispositivo ATR acoplado (FTIR-ATR), análise elementar com microscopia eletrónica de varrimento com energias dispersivas 
de raios X (MEB-EDX) e exame morfológico por técnicas microscópicas de tubos de cofragem como solução oferecida pela indústria da construção para sua utilização como suporte de armazenamento para tapeçarias. Além disso, mostra-se um exemplo prático de uso, empregando-os para o armazenamento da coleção de Tapeçarias de Carmen e Justo Fernández, conservada na Biblioteca Histórica Marquês de Valdecilla da Universidade Complutense.

Palavras-chave: Tapeçarias, armazenamento, conservação preventiva, FTIR-ATR, análises físico-químicas.

\section{Introducción}

Las colecciones de tapices tuvieron su máximo esplendor a partir del siglo XIV, como símbolo de poder y ostentación (López 2017:42), desempeñando un papel muy importante en las distintas manifestaciones de la vida pública y de las celebraciones cortesanas (Herrero 2008: 17). Sin embargo, estas colecciones eran objetos de consumo que se utilizaban con una función determinada. No es hasta mediados del siglo XIX cuando los tapices, y el resto de patrimonio textil empieza a coleccionarse por el simple hecho de ser objetos bellos (Carbonell 2009: 4).

Este pasado como objeto de consumo condicionó la conservación de los tapices en los museos, ya que en un principio continuaron tratándose como si fueran objetos de consumo, por eso se guardaban doblados, apilados unos sobre otros (Herrero 2014: 317).

Este sistema de almacenaje hace años que se abandonó por los problemas de conservación que acarreaba, pues se producían entre otros deterioros, pliegues, deformaciones y tensiones.

Actualmente, se recomienda el almacenamiento de los tejidos en plano, ya que es la forma en que el peso del tejido se reparte por igual, sin generar tensiones ni deformaciones (CCl 2008a).

Este sistema de almacenaje no se adecua a las necesidades de los tapices, debido a sus grandes dimensiones, algunos tapices pueden llegar a medir más de 10 metros de ancho, por lo que el sistema ideal, en el caso de este tipo de obras, es enrollados sobre un soporte cilíndrico (López 2016: 68). El almacenaje de los tapices enrollados sobre un cilindro presenta grandes ventajas, por un lado, permite una manipulación correcta de los tapices, de forma que se evitan posibles deterioros mecánicos (Muñoz-Campos 2004: 74) y por otro, supone un gran ahorro de espacio, ya que existe mobiliario específico, generalmente muebles compactos, para guardar esos soportes cilíndricos, pudiendo colocar unas piezas suspendidas sobre otras, sin afectar a su conservación (López 2013: 34).

Para la construcción de estos soportes cilíndricos se deben emplear materiales químicamente estables, pues la degradación química de estos materiales podría dar lugar a compuestos volátiles que podrían afectar a los tejidos (López 2013: 43). En cuanto a las propiedades físicas del material del soporte, deben tener alta resistencia para poder soportar el peso del tapiz y a la vez, ser ligeros para no incrementar en exceso el peso del conjunto, ya que los tapices pueden alcanzar un elevado peso, lo que dificulta su manipulación.

Los soportes ideales utilizados están fabricados con cartón libre de ácido o polietileno de alta densidad (CCl 2008b). Sin embargo, el elevado precio y la escasa comercialización de los mismos, hace que el uso de estos tubos no sea viable. Por ello, es frecuente recurrir a materiales de construcción, generalmente tuberías de PVC (Policloruro de vinilo) o ABS (Acrilonitrilo butadieno estireno), de fácil acceso en el mercado y con un precio económico. El principal inconveniente derivado del uso de estos materiales como soporte es su elevado peso, lo que se agrava al enrollar el tapiz, además, en el caso del PVC puede experimentar fácilmente una degradación térmica, debido a su estructura química, dando lugar a la formación de estructuras inestables (dobles enlaces conjugados) que pueden intervenir en procesos de oxidación (Yu et al. 2015)

Con respecto al PVC cabe decir que cumple con la rigidez exigible a estos soportes textiles, ya que su temperatura de transición vítrea $(\mathrm{Tg})$ alcanza un valor en torno a $85^{\circ} \mathrm{C}$ lo que hace que en condiciones ambientales normales $\left(20-25^{\circ} \mathrm{C}\right)$ sea rígido y en torno a $90^{\circ} \mathrm{C}$ su comportamiento sea elástico.

Pero se debe indicar que en el procesado de la mayoría de los objetos de PVC, este material polimérico necesita ser aditivado, en primer lugar para rebajar la temperatura de fusión a valores inferiores a $100^{\circ} \mathrm{C}$ y evitar que su degradación térmica se inicie durante su procesado y en segundo lugar para mejorar la elasticidad del producto ya conformado (Shashoua 2008).

Por último, cabe decir que el principal aditivo añadido en las formulaciones de PVC son los plastificantes, en algunos casos puede llegar a superar el 50\% en peso del material (Wilson 1995), además el plastificante se exuda fácilmente, lo que conlleva una disminución de la elasticidad del PVC y una adhesividad residual permanente superficial (García et al. 2008). Estas circunstancias de inestabilidad junto con el elevado peso del material hacen que el PVC no sea un material adecuado para su uso como soporte de almacenaje de tapices.

El ABS es un copolímero termoplástico compuesto por tres monómeros acrilonitrilo, estireno y butadieno, los dos primeros proporcionan resistencia mecánica y rigidez al polímero resultante mientras que el tercero aporta ductilidad a baja temperatura, esto hace que el ABS sea un material con buenas propiedades de resistencia mecánica además de liviano, pero el problema radica 
en la mala capacidad para ser procesado por lo que las modificaciones realizadas en el procesado del material hacen que se encarezca el producto.

Por todo lo expuesto, es necesario buscar alternativas para soportes cilíndricos de almacenaje que estén compuestos por materiales estables, que sean ligeros y, por último, accesibles en el mercado y con un precio asequible. La industria de la construcción ofrece otras soluciones que pueden ser valoradas para su utilización como soporte de almacenaje para tapices: los tubos para encofrado [Figura 1]. Estos tubos son ligeros, y fácilmente accesibles en el mercado y con un bajo coste. Antes de su uso como material de conservación se debe realizar un estudio de los materiales que componen los tubos de encofrado para determinar sus características morfológicas y su composición química y así verificar su estabilidad química y por lo tanto su compatibilidad con los tapices.

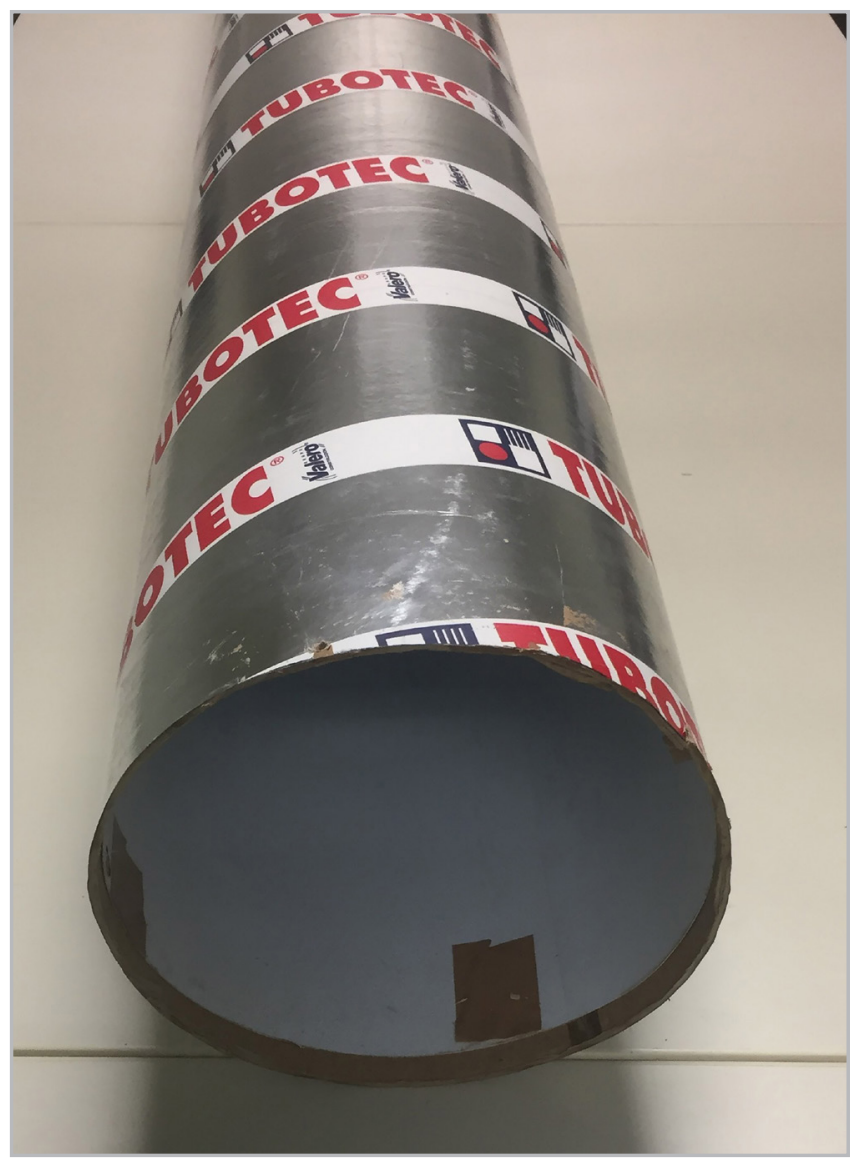

Figura 1.- Tubo de encofrado

\section{Objetivo}

El objetivo de esta investigación es el estudio de la composición química mediante FTIR-ATR, SEM-EDX y el examen morfológico por microscopía estereoscópica de los materiales que componen los tubos de encofrado, además de la aplicación de estos como soporte de almacenaje para una obra real.

\section{Metodología}

La metodología seguida ha sido aplicada en sucesivas etapas.

La primera comprende el estudio de la información comercial. Para ello se han consultado las páginas web y los catálogos de productos de diferentes proveedores de material de la construcción (Encocentro, Aislenvas, Fatecsa y Valero).

En una segunda etapa se ha procedido al análisis de los tubos de encofrado, estudiando dos tubos de diferentes marcas comerciales, Tubotec ${ }^{\circledR}$ (suministrado por Grupo Valero) y $\mathrm{TBT}^{\circledR}$ espiral (suministrado por Encocentro), en el Laboratorio de Materiales de la Facultad de Bellas Artes de la Universidad Complutense de Madrid [LabMat] y el Centro Nacional de Microscopía Electrónica (ICTS) de la Universidad Complutense de Madrid.

Para el estudio de la composición y estructura de los materiales se ha seguido el protocolo instaurado en el [LabMat] para el estudio de materiales poliméricos utilizados en conservación y restauración de bienes culturales (Chércoles 2015).

Se ha realizado un examen microscópico para determinar la morfología de material, para ello se ha utilizado un microscopio estereoscópico modelo Leica MZ 125 equipado con iluminador de luz fluorescente y una cámara digital Leica DC150. Para este tipo de examen no es necesaria la preparación previa de la muestra, se ha tomado un fragmento del tubo de encofrado asegurándose que fuera completo (con todas las capas presentes) y se ha procedido a su observación.

La técnica analítica de espectroscopía infrarroja por transformada de Fourier en modo ATR (Reflectancia Total Atenuada) se emplea habitualmente en el estudio de polímeros y de los materiales resultantes de su procesado (Chércoles et al. 2009). Su aplicación tiene como finalidad identificar la composición química del material y estudiar su morfología. Para ello se ha empleado un equipo Thermo Nicolet 380, con detector DTGS/KBr, cubriendo un rango de 4000 a $400 \mathrm{~cm}^{-1}$. Los espectros fueron recogidos en modo absorbancia después de 64 barridos y con una resolución espectral de $4 \mathrm{~cm}^{-1}$. Se ha empleado un accesorio de reflexión total atenuada (ATR) de cristal de diamante. Los espectros han sido analizados utilizando el software Omnic v 7.3.

La espectroscopia FTIR-ATR presenta ciertas peculiaridades, entre las que destaca la ventaja de no ser necesaria la preparación previa de la muestra. Asimismo, hay que señalar que el análisis realizado con FTIR-ATR es superficial, circunstancia que se debe tener en cuenta en el caso de materiales que están constituidos por la superposición de diferentes materiales como es el caso de los tubos de encofrado. Debido a esto, en primer lugar se ha procedido 
a separar cada una de las capas presentes en el tubo y realizándose el análisis sobre cada una de ellas, tanto en su cara externa como en la interna.

Para asegurar la reproducibilidad de las medidas se han recogido tres espectros para cada una de las capas del material del tubo (San Andrés et al. 2010). Para determinar la composición de una de las capas de aspecto metálico se ha procedido a realizar un análisis elemental sobre dicha capa para su caracterización con un microscopio electrónico de barrido JEOL 6400 JSM, con análisis elemental cualitativo con una resolución de $133 \mathrm{eV}$.

Por último, en una tercera etapa se ha procedido a la aplicación directa sobre un caso real, en este caso la construcción de soportes de almacenaje para la Colección de Tapices de Carmen y Justo Fernández en la Biblioteca Histórica Marqués de Valdecilla de la UCM [Figura 2].

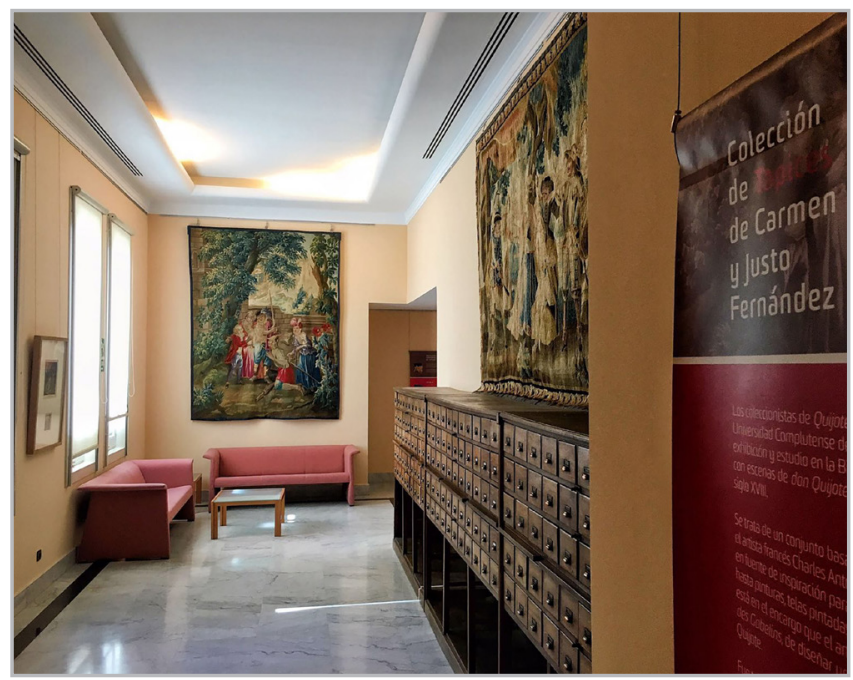

Figura 2.- Vista de la sala de tapices de la Biblioteca Histórica Marqués de Valdecilla de la Universidad Complutense de Madrid.

\section{Resultados}

Se han estudiado los tubos de encofrado de las marcas comerciales Tubotec $^{\circledast}$ y TBT $^{\circledR}$ espiral, se ha comprobado que ambos están fabricados con los mismos materiales por lo que se presentarán los resultados de uno de ellos, en concreto del tubo $\mathrm{TBT}^{\circledR}$ espiral.

El examen microscópico aporta información sobre la estructura multicapa de los tubos, tipo tetra pack ${ }^{\circledR}$ (Tetra pack 2020). Este material está formado por cinco capas superpuestas, tal y como indica el fabricante, del exterior al interior, una capa fina transparente de aspecto plástico (capa 1), una capa de color gris brillante (capa 2), dos capas de material fibroso, una de color marrón (capa 3) y otra de color blanco (capa 4) y una última capa de color blanco con aspecto plástico (capa 5). Está secuencia se repite cinco veces más [Figura 3].

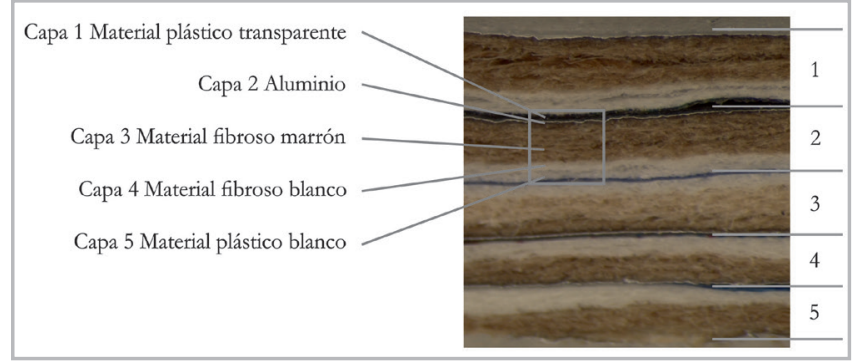

Figura 3.- Imagen con el microscopio estereoscópico de las capas.

Las técnicas microscópicas también aportan información sobre las características morfológicas constatando diferencias significativas en cuanto al procesado de los diferentes materiales que forman las capas, se puede decir que el tubo está compuesto por tres tipos de materiales procesados de diferente forma: material laminado con bajo espesor (film) (capas 1 y 5), una lámina de aspecto metálico, la capa 2 [Figura 4a] y por último material fibroso (capas 3 y 4) [Figura 4b].
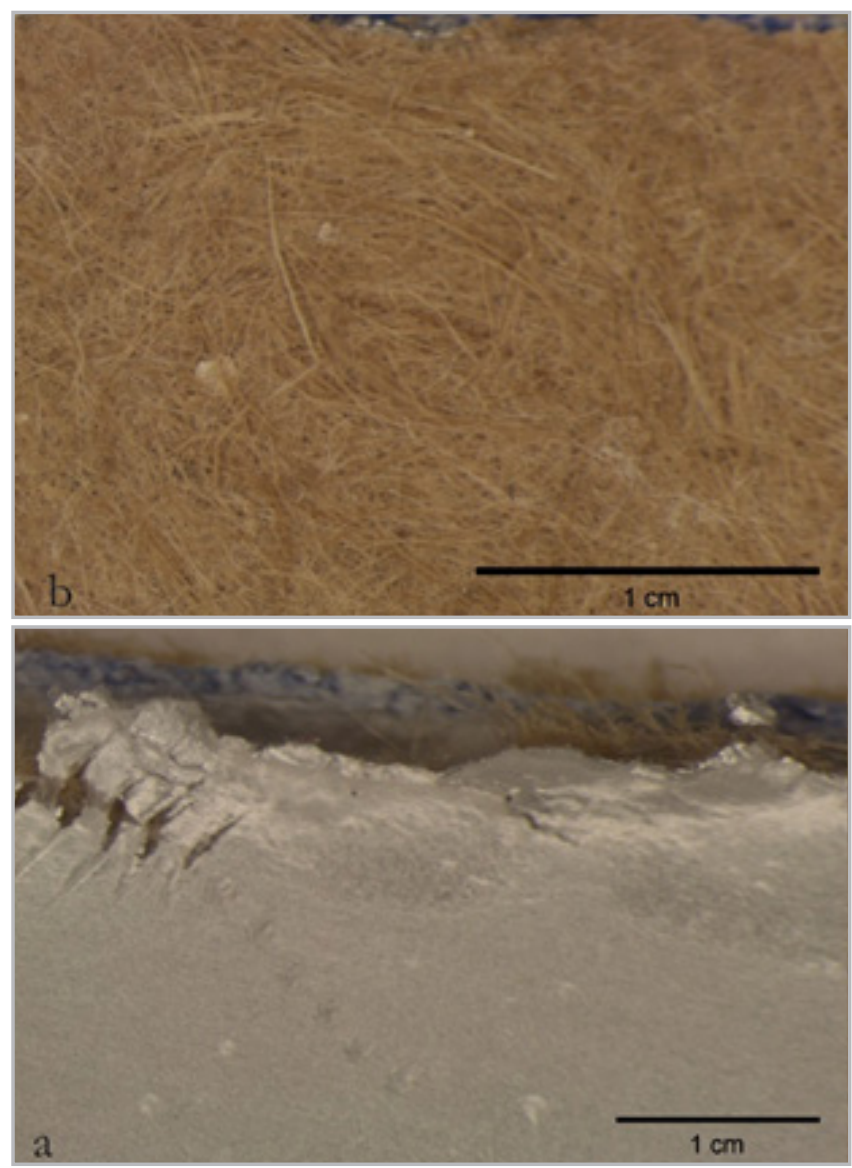

Figura 4.- Imagen en detalle de las capas de polietileno y aluminio (a) y detalle del material fibroso marrón (b).

La composición química de las distintas capas viene determinada por los análisis por FTIR-ATR.

La identificación de los distintos componentes se ha realizado mediante la asignación de las bandas a 
determinados grupos químicos y por comparación de los espectros de las muestras con librerías de patrones de referencia (Socrates 2001, IRUG 2020, Hummel library 2021).

Los espectros FTIR-ATR de las capas 1 y 5 son idénticos y las bandas de absorción presentes corresponden con la vibración del grupo metileno $(-\mathrm{CH} 2-)\left(\mathrm{v}_{\mathrm{as}} 2914 \mathrm{~cm}^{-1} ; \mathrm{v}_{\mathrm{s}}\right.$ $2847 \mathrm{~cm}^{-1}, \delta$ y $\delta_{\mathrm{s}}$ en el plano $1464 \mathrm{~cm}^{-1}$ ) y con las bandas de vibración de deformación que aparecen dentro del intervalo $720-710 \mathrm{~cm}^{-1}$, en concreto a $718 \mathrm{~cm}^{-1}$ (San Andrés et al. 2013). Estas bandas son características del polietileno de baja densidad [Figura 5].

En los espectros de las capas 3 y 4 se pueden asignar las siguientes bandas que corresponden con la celulosa: $\mathrm{vOH}$ $\left(3321\right.$ y $\left.3290 \mathrm{~cm}^{-1}\right), \delta_{\text {en el plano }} \mathrm{OH}(1453,1315,1204$ y 658 $\left.\mathrm{cm}^{-1}\right), \mathrm{v}_{\text {as }} \operatorname{COC}\left(1157 \mathrm{~cm}^{-1}\right)$. También han sido identificadas $\mathrm{s}$ bandas correspondientes al carbonato cálcico $\left(\mathrm{CaCO}_{3}\right): \mathrm{v}_{\mathrm{as}}$
C-O $\left(1423 \mathrm{~cm}^{-1}\right), \delta_{\text {fuera del plano }} \mathrm{C}-\mathrm{O}\left(896 \mathrm{~cm}^{-1}\right)$ y $\delta_{\text {en el plano }}$ $\left(710 \mathrm{~cm}^{-1}\right)$. (San Andrés et al. 2010). [Figura 6].

Se debe mencionar que la celulosa y el polietileno son los materiales mayoritarios presentes y aunque se han podido detectar la presencia de carbonato cálcico como aditivo en las capas 1 y 5, para conocer la presencia de otros componentes minoritarios como aditivos, se deben realizar otros análisis capaces de detectar su presencia como la pirolisiscromatografía de gases-espectrometría de masas, en otros estudios realizados los principales componentes minoritarios presentes en las capas de polietileno de baja densidad son aditivos derivados del ácido ftálico (Chércoles 2015).

En cuanto a la composición de la capa 2, de aspecto metálico, una vez separada del resto de capas se ha observado la superficie y se ha realizado un análisis elemental para determinar su composición, tal y como se observa en el espectro EDX se determinó la presencia de aluminio [Figura 7].

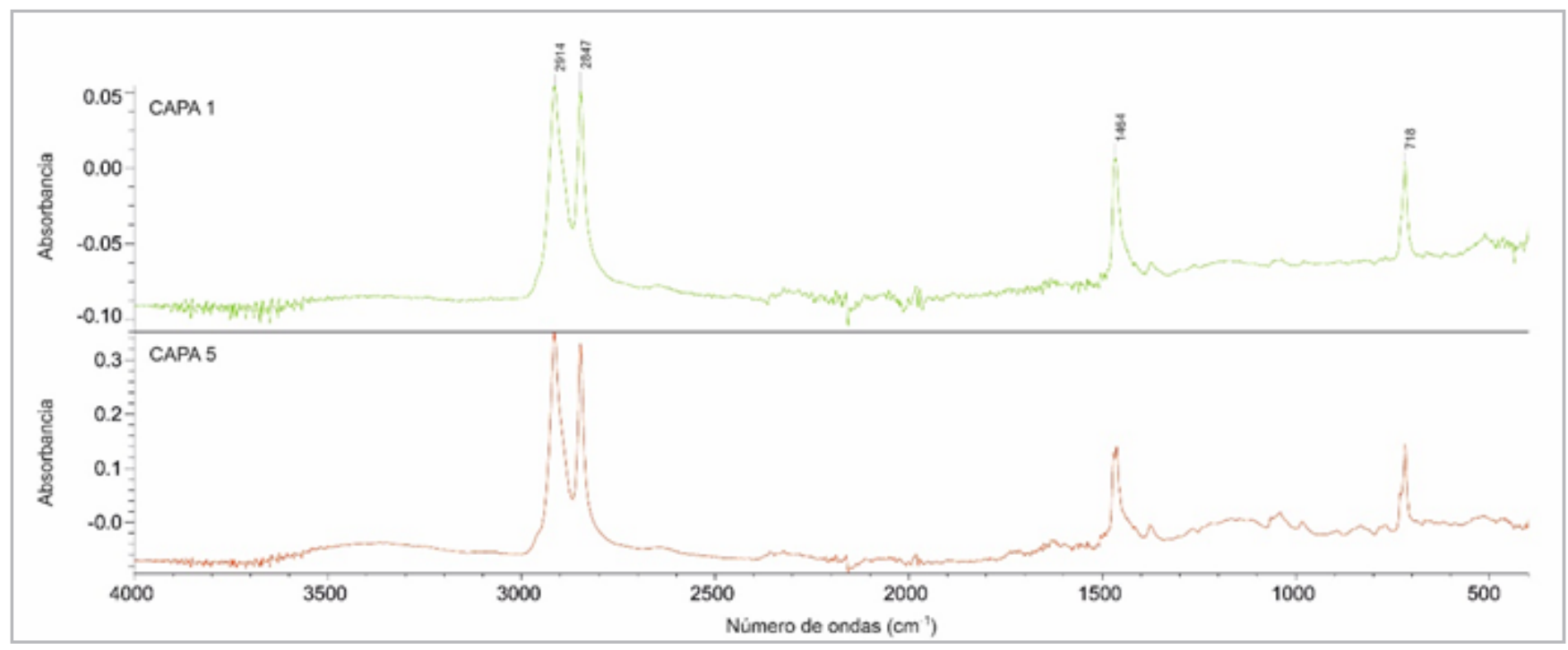

Figura 5.- Espectros FTIR-ATR de las capas 1 y 5, polietileno de baja densidad.

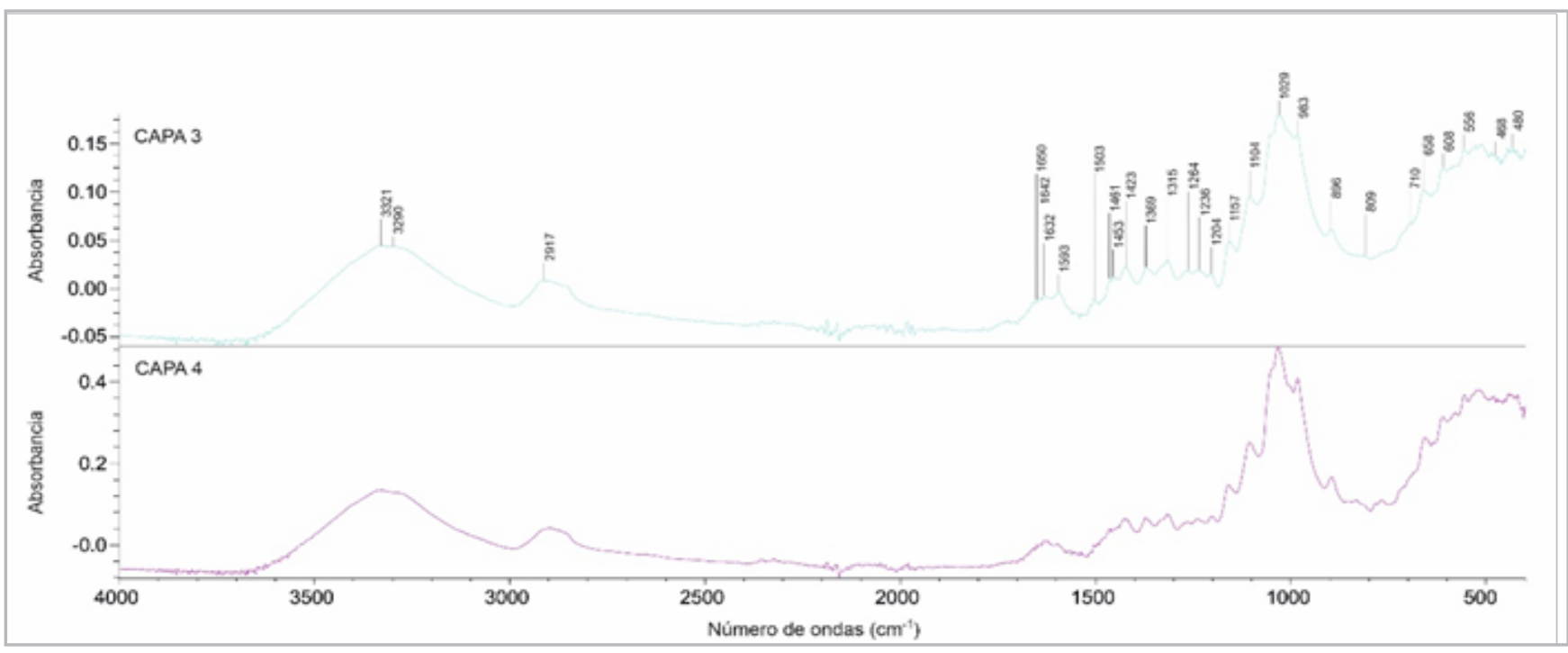

Figura 6.- Espectros FTIR-ATR de las capas 3 y 4, celulosa. 


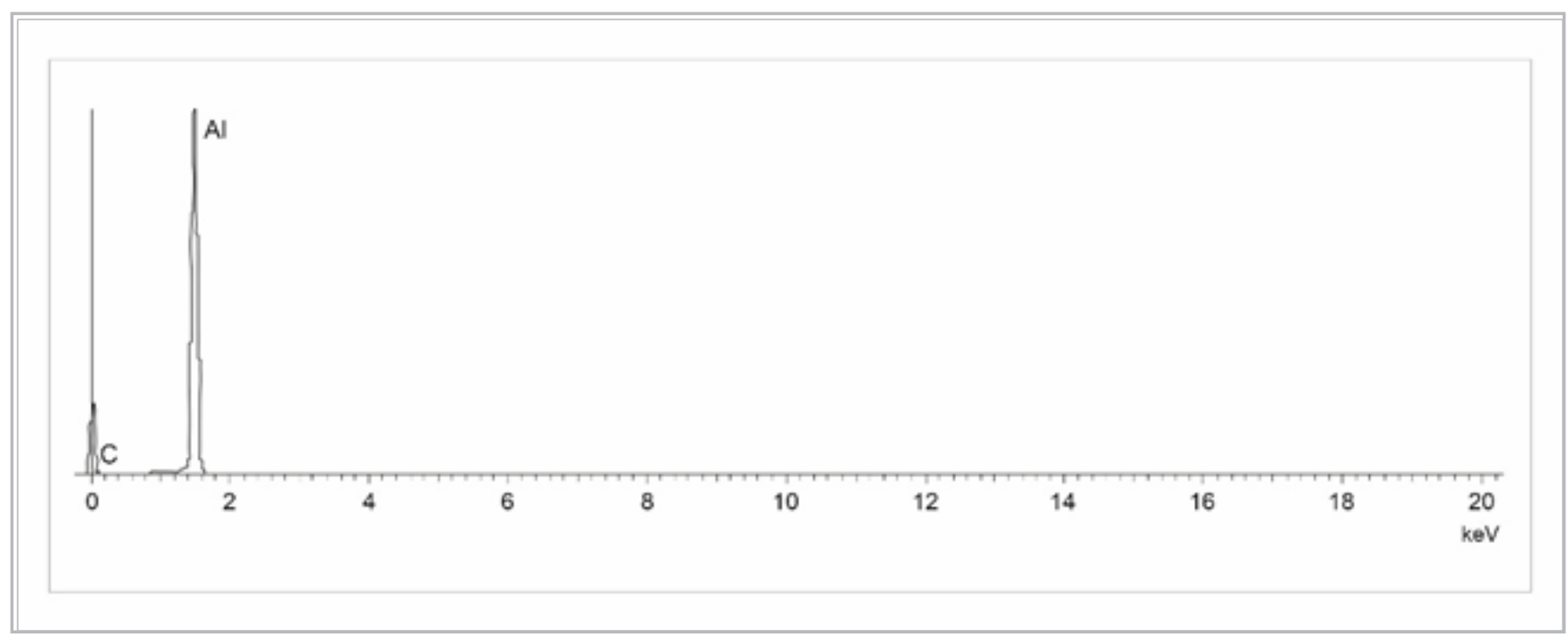

Figura 7.- Espectro EDX de la capa 2, aluminio.

La revisión de la información comercial expuesta en los catálogos de materiales de construcción y en las fichas técnicas de los tubos se especifica que los tubos de encofrado están fabricados mediante un proceso de termosoldado helicoidal de capas alternativas de papel Kraft, aluminio y polietileno (KAP) en relación 1:1:4. Es un material multicapa (un total de 6) dónde la sucesión de las mismas es la siguiente: polietileno con densidad de 13 $\mathrm{gr} / \mathrm{m}^{2}$, polietileno con densidad de $12 \mathrm{gr} / \mathrm{m}^{2}$, aluminio de $22 \mathrm{gr} / \mathrm{m}^{2}$ de densidad, polietileno con densidad de 12gr/ $\mathrm{m}^{2}$, papel Kraft de fibra larga de $12 \mathrm{gr} / \mathrm{m}^{2}$ de densidad y una última capa de polietileno con densidad de $13 \mathrm{gr} /$ $\mathrm{m}^{2}$ (Aislenvas 2019: 73). La unión de las distintas capas se realiza mediante la fusión del polietileno (Encocentro 2020). Los tubos se suministran con una longitud máxima de 4 metros y con diámetros desde los $15 \mathrm{~cm}$ a los $120 \mathrm{~cm}$ (Aislenvas 2019: 73). Por último, se constata la ligereza de los tubos, que pesan entre un $1 \mathrm{Kg}$ por cada metro lineal de tubo, en el caso del tubo con $15 \mathrm{~cm}$ de diámetro y los $6 \mathrm{~kg}$ por cada metro lineal del tubo de $60 \mathrm{~cm}$ de diámetro (Valero 2019).

Los resultados de composición química obtenidos corresponden al material Tetrapack ${ }^{\circledast}$ (Tetrapack 2020) y corrobora la información ofrecida por los distribuidores de los tubos de encofrado.

Por último se exponen los resultados y el proceso de la preparación de tubos de encofrado para usarlos como soporte de almacenamiento en los tapices de la Colección de Tapices de Carmen y Justo Fernández.

La Colección de Tapices de Carmen y Justo Fernández fue depositada bajo la fórmula de comodato por sus propietarios. Está formada por cuatro tapices (Las Bodas de Camacho, Quijote armado caballero, El Rucio de Sancho, La Princesa Micomicona) y un tejido bordado (Entrada del Amor y la Riqueza en las Bodas de Camacho) (López 2020: 68). Dentro del programa de conservación preventiva establecido en la Biblioteca Histórica, se alternan periodos de exposición con periodos de almacenaje en el depósito de la biblioteca, que cuenta con las condiciones climáticas y de seguridad adecuadas para la conservación de patrimonio cultural (López 2020: 73). Para el almacenamiento de los tapices se construyeron cinco soportes cilíndricos, adecuados a las medidas y necesidades de cada uno de los tapices, que además facilitaban su manipulación en los movimientos internos dentro de la biblioteca [Figura 8].

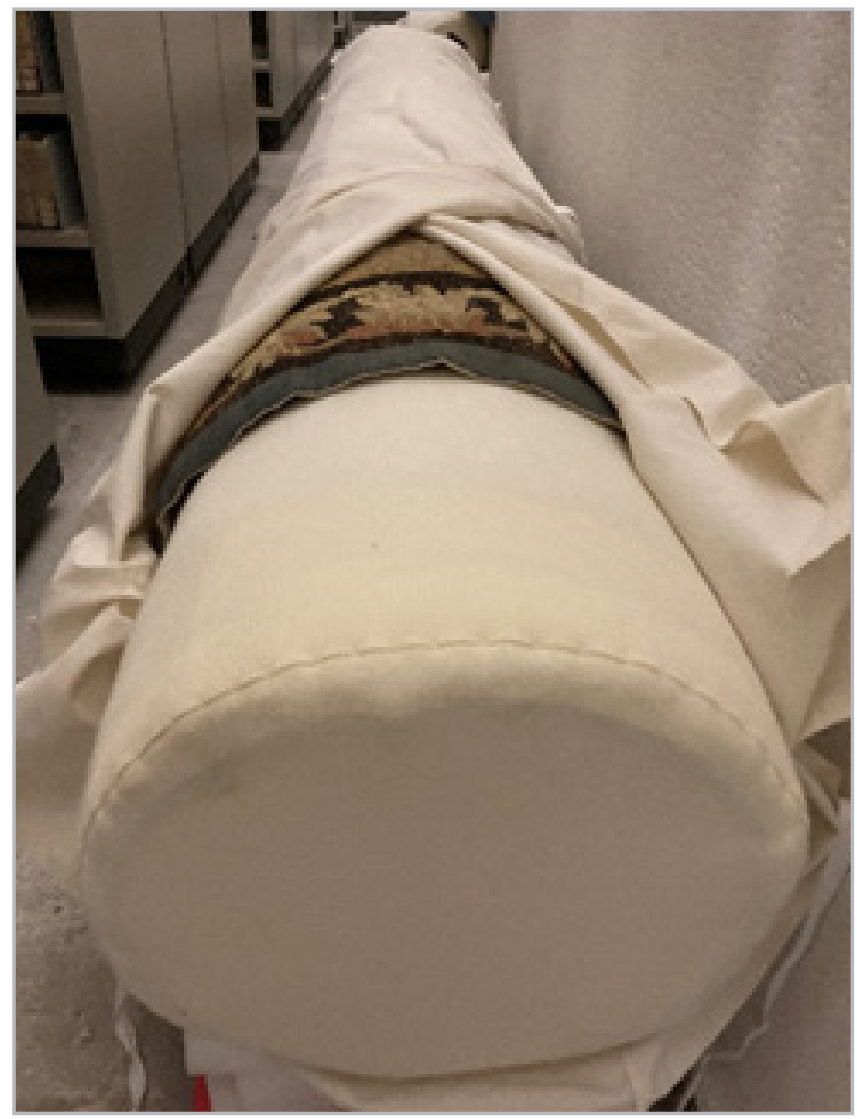

Figura 8.- Tapiz de Las Bodas de Camacho en su soporte de almacenaje. @ Fotografía de Javier Tacón. 
Para la construcción de los cinco soportes de almacenaje se emplearon tubos de encofrado, de dos marcas comerciales diferentes, pero, tal y como se ha mencionado anteriormente, con idéntica composición química y estructura. Se seleccionaron tres de la marca TBT $^{\circledR}$ espiral y dos de la marca Tubotec ${ }^{\circledast}$. Los tubos de la marca TBT ${ }^{\oplus}$ tienen $25 \mathrm{~cm}$ de diámetro mientras que los de Tubotec ${ }^{\circledR}$ tienen $30 \mathrm{~cm}$, en ambos casos tienen un diámetro suficiente para minimizar la curvatura al enrollar el tapiz (Carbonell y Cerdà 2011: 13). Los tubos tienen una longitud máxima de 4 metros, lo que permite construir los soportes de una sola pieza. En este caso la longitud de cada tubo se determinó añadiendo 40 centímetros a la altura del tapiz, tomando esta como referencia, teniendo en cuenta que siempre se enrollan en sentido de la urdimbre (López 2019: 37).

El primer paso ha sido el acolchado del tubo con guata de poliéster (tejido no tejido de poliéster), y forrado con tejido tubular de algodón de punto elástico (ventulón ${ }^{\circledR}$ ) [Figura 9].

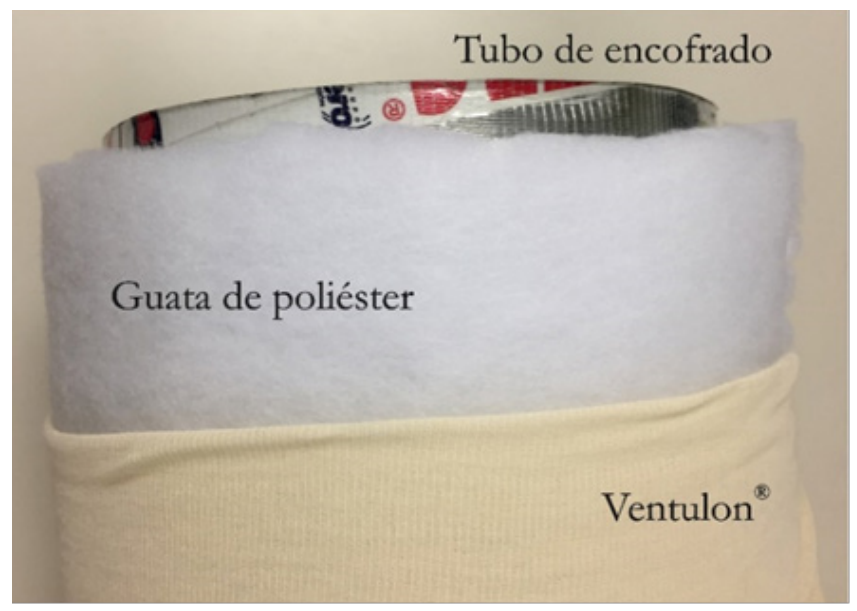

Figura 9.- Soporte de almacenaje para tapices

El principal objetivo de este acolchado es preparar la superficie del tubo para recibir el tapiz, facilitando su enrollado. En cuanto a la guata de poliéster cabe decir que la morfología de las fibras de poliéster, tubular y con un entramado irregular sin compactar, característica de las fibras de origen sintético, proporcionan un filtro almohadillado que resulta excelente como forro de embalajes para actuar como amortiguador y protector frente a vibraciones mecánicas. El forrado con ventulón ${ }^{\circledR}$ proporciona una superficie lisa para el tapiz, y es fácilmente sustituible cuando este se deteriora o ensucia. Además, con este acolchado y forrado se aísla el material del tubo del tapiz, evitando las posibles emisiones de la degradación oxidativa de las capas de cartón (pastas mecánicas).

Para cerrar los extremos del tubo y para fabricar unas bases sobre las que descanse el tubo se ha utilizado espuma de polietileno de baja densidad, (Ethafoam ${ }^{\circledast}$ ) material celular, dónde la presencia de células cerradas hace que sea un material apropiado como protector frente al impacto $y$ las vibraciones, aislante térmico y de humedad, además la baja densidad del material le proporciona ligereza (Chércoles 2015: 264). Todo ello unido a que al cerrar los tubos se evita la entrada de polvo y suciedad en el interior del mismo [Figura 10a].

Además, se han construido unas bases de Ethafoam ${ }^{\circledR}$ sobre las que descansan los soportes de almacenaje de forma que el tapiz enrollado nunca soporta su propio peso en la zona de apoyo [Figura 10b]. Estas bases siempre se colocan en los extremos del soporte, y nunca deben entrar contacto con el tapiz pues generarían tensiones en las zonas de contacto. Debemos recordar que el tubo es suficientemente largo y el tapiz no llega a los extremos.

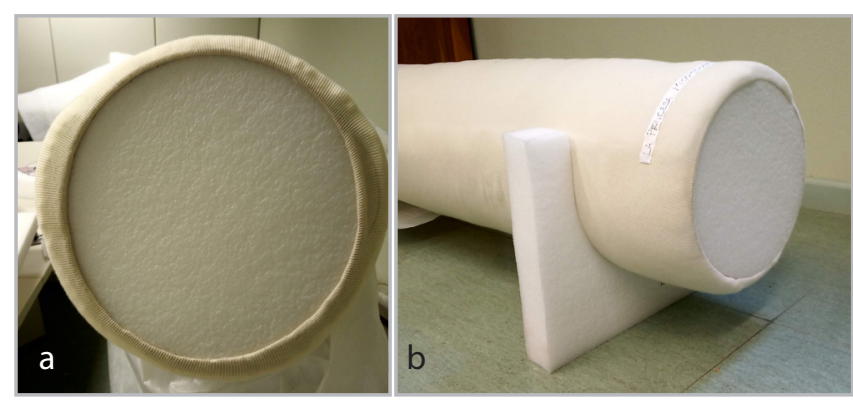

Figura 10.- Detalle de los extremos del soporte (a) y de las bases de ethafoam ${ }^{\circledast}(b)$.

\section{Conclusiones}

Es necesario realizar análisis previos de materiales antes de su uso en conservación para comprobar la composición química de los mismos y evaluar su compatibilidad con las obras de arte que estén en contacto.

Los análisis por FTIR-ATR, SEM-EDX y examen microscópico corroboran los resultados ofrecidos por el fabricante, dónde los materiales mayoritarios son polietileno $y$ celulosa y el material es multicapa.

Además, el material multicapa aporta la resistencia necesaria para soportar el peso del tapiz, y posee a la vez ligereza, lo que favorece la manipulación del soporte, incluso con el tapiz enrollado.

Los tubos de encofrado son un material accesible, existen numerosos distribuidores de este tipo de material, y tienen un precio muy económico.

Es necesario el acolchado del tubo con guata de poliéster y el forrado con Ventulón ${ }^{\circledR}$ para facilitar el enrollado y de paso, aislar el material del tubo del tapiz. Además, es recomendable cerrar los extremos del tubo con Ethafoam ${ }^{\circledR}$ para evitar la entrada de suciedad en su interior. Para repartir el peso del soporte con el tapiz enrollado y evitar que el conjunto toque el suelo y soporte su propio peso, se disponen dos bases de Ethafoam ${ }^{\circledR}$ en los extremos del tubo, evitando que estas bases estén en contacto con el tapiz. 
Por último con la aplicación práctica de los tubos de encofrado como soporte de almacenaje para el enrollado de tapices en el almacenaje se ha constatado que ofrecen una buena solución como alternativa a otros materiales.

Como línea de investigación futura, para ampliar el conocimiento de la composición de los tubos de encofrado se propone la realización de análisis de Pirólisis-Cromatografía de gases-Espectrometría de masas que permita identificar los componentes minoritarios presentes en cada una de las capas. Además, para conocer el comportamiento a largo plazo del material se propone diseñar una metodología específica de envejecimiento artificial acelerado bajo variables controladas y evaluar los cambios producidos en su composición a los diferentes tiempos de envejecimiento.

\section{Referencias}

AISLENVAS (2019). Aislamientos y complementos para la construcción. Disponible en: https://www.aislenvas.es/wpcontent/uploads/2019/09/catalogo-almacenes-y-construccion. pdf [consulta: 18/12/2020].

CANADIAN CONSERVATION INSTITUTE (2008a). Flat storage for Textiles, CCI Note 13/2. Disponible en: https://www.canada.ca/ en/conservation-institute/services/conservation-preservationpublications/canadian-conservation-institute-notes/flat-storagetextiles.html [consulta: 20/9/2020].

CANADIAN CONSERVATION INSTITUTE (2008b). Rolled Storage for Textiles, CCI Note 13/3. Disponible en: https://www.canada.ca/ en/conservation-institute/services/conservation-preservationpublications/canadian-conservation-institute-notes/rolledstorage-textiles.html [consulta: 20/9/2020].

CARBONELL BASTÉ, S. (2009). “Los inicios del coleccionismo Textil en Cataluña". Datatextil, 21: 4-27.

CARBONELL BASTÉ, S. y CERDÀ DURÀ, E. (2011) “Criterios básicos sobre la manipulación de los objetos textiles patrimoniales". En Manipulación, almacenaje y transporte de material textil. Terrassa: Centro de Documentación y Museo Textil de Terrassa, 7-18.

CHÉRCOLES, R. (2015). Estudio del comportamiento físico-químico de materiales poliméricos utilizados en conservación y restauración de bienes culturales. Tesis Doctoral, Universidad Complutense de Madrid, Madrid.

CHÉRCOLES, R; SAN ANDRÉS, M.; DE LA ROJA, J.M. y GÓMEZ, M.L. (2009).“Analytical Characterization of Polymers used in Conservation and Restoration by ATR-FTIR." Analytical and Bioanalytical Chemistry, 395: 2082-2096. https://doi.org/10.1007/s00216-009-3201-2

ENCOCENTRO (2020). TBT circular. Disponible en: https://www. encocentro.com/portfolio/tbt-circular/ [consulta: 18/12/2020].

FATECSA (2019). Encofrado "TBT" (Construcción). Disponible en: https://fatecsa.com/productos/encofradotbtconstruccion/ [consulta: 18/12/2020].

GARCÍA FERNÁNDEZ-VILLA, S.; DE LA ROJA, J.M. y SAN ANDRÉS, M. (2008), "Procesos y efectos de la degradación del PVC plastificado". En 9a Jornadas de Conservación de Arte Contemporáneo. Madrid: Museo Nacional Centro de Arte Reina Sofía, 47-59.

HERRERO CARRETERO, C. (2008). Vocabulario Histórico de la Tapicería. Madrid, Patrimonio Nacional.

HERRERO CARRETERO, C. (2014). "Sistemas expositivos de tapices y textiles. Colección de Patrimonio Nacional: logros y propuestas". Anales de Historia del Arte, 24: 307-326. http://dx.doi.org/10.5209/ rev ANHA.2014.48280

The Hummel Polymers and Additives Library Hummel Polymers and Additives FTIR Spectral Library.

IRUG (2021). Infrared \& Raman Users Group. Disponible en: http:// www.irug.org [consulta: 19/01/2021].

LÓPEZ REY, M. (2013). Conservación Preventiva de colecciones textiles. Avilés: ARA, Asociación Profesional de Conservadores y Restauradores del Principado de Asturias.

LÓPEZ REY, M. (2016). "Aproximación a la conservaciónrestauración de los tapices", Pecia Complutense, 24: 60-69. https:// eprints.ucm.es/id/eprint/35661/7/Pecia24-4.pdf

LÓPEZ REY, M. (2017). Métodos y materiales de limpieza alternativos al medio acuoso en tratamientos de conservación-restauración de materiales textiles. Tesis Doctoral, Universidad Complutense de Madrid, Madrid. https://eprints.ucm.es/id/eprint/48880/1/T40415.pdf

LÓPEZ REY, M. (2019). "Memoria del Proceso de Conservaciónrestauración El Rucio de Sancho", Documentos de trabajo UCM. Biblioteca Histórica, 2019/02. Disponible en: https://eprints.ucm. es/51166/2/DT2019-02.pdf [consulta: 18/12/2020].

LÓPEZ REY, M. (2020). "Tapestry Collection at the Biblioteca Histórica Marqués de Valdecilla, Complutense University of Madrid", Datatèxtil, 40: 65-74.

MUÑOZ-CAMPOS, P. (2004). "Conservación y almacenamiento de tejidos: problemas múltiples, soluciones prácticas", Museos.es: Revista de la Subdirección General de Museos Estatales, 0: 72-79.

SHASHOUA, Y. (2008). Conservation of Plastics. Material Science, Degradation and Preservation, Oxford: Butterworth Heineman.

SAN ANDRÉS, M.; de la ROJA J.M.; CHÉRCOLES, R.; GÓMEZ, M. y BAONZA, V.G. (2010): “Envejecimiento con radiación UV de un cartón pluma neutro. Estudio de su evolución cromática y composición". Óptica Pura y Aplicada, 43 (4): 219-227.

SAN ANDRÉS, M.; CHÉRCOLES, R.; GÓMEZ, M.L. y DE LA ROJA, J.M. (2010). "Materiales sintéticos utilizados en la manipulación, exposición y almacenamiento de Obras de Arte y Bienes 
Culturales. Caracterización por espectroscopía FTIR-ATR". En $11^{a}$ Jornada de Conservación de Arte Contemporáneo. Madrid: Museo Nacional Centro de Arte Reina Sofía, 33-51.

SAN ANDRÉS, M., CHÉRCOLES, R., SANTOS, S., DE LA ROJA, J.M., DOMÍNGUEZ, C.Y GÓMEZ, M. (2013). "Materials made from Polyolefins used in tasks of Preventive Conservation. A comparative study of their long-term behavior". En M. A. Rogerio, M. Lazzari y E. Cano, (eds), Science and Technology for the Conservation of Cultural Heritage. Londres: Taylor \& Francis Group, 341-344.

SOCRATES, G. (2001). Infrared and Raman Characteristic Group Frequencies, Tables and Charts. Los Ángeles: Wiley.

TETRA PAK (n.d.). Material para envasado para envases de cartón Tetra Pak. Disponible en https://www.tetrapak.com/es-es/ solutions/packaging/packaging-material/materials [consulta: 18/12/2020].

VALERO (n.d). Tubotec. Disponible en: https://www.grupovalero. com/productos/soluciones-constructivas/encofrados/tubotec/ [consulta: 18/12/2020].

VALERO (2019). Ficha técnica Tubotec. Disponible en: https:// www.grupovalero.com/productos/soluciones-constructivas/ encofrados/tubotec/ [consulta: 18/12/2020].

WILSON, A. S. (1995). Plasticisers: Principles and Practice, Londres: The Institute of Materials.

YU, J.; SUN, L.; MA CH.; QIAO, Y.; YAO, H. (2015): “Thermal degradation of PVC: A review", Composites Science and Tecnology, 117: 398-403. https://doi.org/10.1016/j.wasman.2015.11.041

\section{Autor/es}

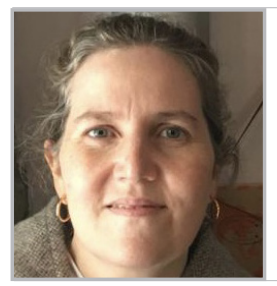

\section{María López Rey}

marialopezrey@yahoo.es

Escuela Superior de Arte del Principado de Asturias

https://orcid.org/0000-0001-8578-5182

Licenciada en Bellas Artes por la Universidad Politécnica de Valencia y Máster en Proyectos de Conservación, restauración y Doctora en Bellas Artes por la Universidad Complutense de Madrid. Su actividad profesional, investigadora y docente está vinculada a la Conservación y Restauración del Patrimonio Cultura desde hace 17 años. Centrándose en el campo de la conservación y restauración de materiales textiles. Como conservadora-restauradora de materiales textiles ha trabajado en diferentes instituciones públicas $y$ privadas como el Centro de Documentación y Museo Textil de Terrassa, el Museo Cerralbo, la Fundación Balenciaga y la Fundación Princesa de Asturias, entre otros. Ha sido profesora en Grado de Conservación y Restauración de la Facultad de Bellas Artes de la UCM y actualmente es profesora en el Título Superior de Conservación y Restauración en la Escuela Superior de Arte del Principado de Asturias. Su labor investigadora se centra en la conservación-restauración de los materiales textiles, con estancias en el Instituto Andaluz del PatrimonioHistórico,enellaboratoriodematerialesdelaFacultadde Bellas Artes de la UCM, y en el Metropolitan Museum de Nueva York. Esta trayectoria ha dado lugar a publicaciones y a la participación en diferentes congresos nacionales e internacionales además de la impartición de varios cursos relacionados con el ámbito de Conservación y Restauración de materiales textiles.

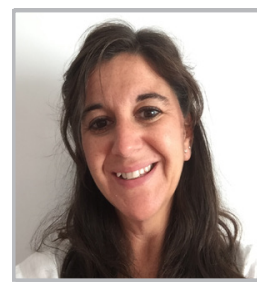

\section{Ruth Chércoles Asensio}

rmcherco@ucm.es

Universidad Complutense de Madrid https://orcid.org/0000-0002-0689-4266

Licenciada en Ciencias Químicas y Doctora en Bellas Artes por la Universidad Complutense de Madrid, Máster de Restauración y Rehabilitación de Patrimonio Histórico de la Universidad de Alcalá de Henares. Su actividad investigadora, docente y profesional está vinculada a la Conservación y Restauración del Patrimonio Cultura desde hace 17 años, especializándose en el estudio de propiedades, comportamiento y técnicas de análisis aplicadas a materiales del patrimonio cultural. Coordinadora y profesora en el Grado de Conservación y Restauración de la Facultad de Bellas Artes de la UCMy Responsable de Calidad del laboratorio de materiales de la Facultad de Bellas Artes de la UCM, incluido dentro de la Red de laboratorios e Infraestructuras de la Comunidad Autónoma de Madrid. Su labor investigadora en los últimos años se centra en la caracterización de polímeros utilizados en conservación y restauración y el estudio de su comportamiento físico y químico aplicando ensayos de envejecimiento artificial. Esta trayectoria ha dado lugar a publicaciones y a la participación en diferentes congresos nacionales e internacionales además de la impartición de varios cursos y másteres relacionados con el ámbito de la ciencia aplicada a la Conservación y Restauración.

Artículo enviado el 07/09/2021

Artículo aceptado el 19/12/2021

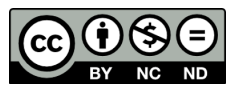

https://doi.org/10.37558/gec.v21i1.1043 